\title{
Nonadherence in the era of severe asthma biologics and thermoplasty
}

\author{
Joy Lee ${ }^{1,2}$, Tunn Ren Tay ${ }^{1}$, Naghmeh Radhakrishna ${ }^{1}$, Fiona Hore-Lacy ${ }^{1}$, \\ Anna Mackay ${ }^{1}$, Ryan Hoy ${ }^{1,2}$, Eli Dabscheck ${ }^{1,3}$, Robyn O'Hehir ${ }^{1,3}$ and Mark Hew ${ }^{1,2}$
}

Affiliations: ${ }^{1}$ Allergy, Asthma and Clinical Immunology, The Alfred Hospital, Melbourne, Australia. ${ }^{2}$ School of Public Health and Preventive Medicine, Monash University, Melbourne, Australia. ${ }^{3}$ Allergy, Immunology and Respiratory Medicine, Central Clinical School, Monash University, Melbourne, Australia.

Correspondence: Joy Lee, Allergy, Asthma and Clinical Immunology, The Alfred hospital, 55 Commercial Road, Melbourne, Victoria 3181, Australia. E-mail: joy.leedalfred.org.au

@ERSpublications

Preventer adherence is underrecognised and must be confirmed objectively prior to initiating novel asthma treatment http://ow.ly/Kc1X30iysYD

Cite this article as: Lee J, Tay TR, Radhakrishna N, et al. Nonadherence in the era of severe asthma biologics and thermoplasty. Eur Respir J 2018; 51: 1701836 [https://doi.org/10.1183/13993003.01836-2017].

ABSTRACT Nonadherence to inhaled preventers impairs asthma control. Electronic monitoring devices (EMDs) can objectively measure adherence. Their use has not been reported in difficult asthma patients potentially suitable for novel therapies, i.e. biologics and bronchial thermoplasty.

Consecutive patients with difficult asthma were assessed for eligibility for novel therapies. Medication adherence, defined as taking $>75 \%$ of prescribed doses, was assessed by EMD and compared with standardised clinician assessment over an 8-week period.

Among 69 difficult asthma patients, adherence could not be analysed in 13, due to device incompatibility or malfunction. Nonadherence was confirmed in 20 out of 45 (44.4\%) patients. Clinical assessment of nonadherence was insensitive (physician 15\%, nurse 28\%). Serum eosinophils were higher in nonadherent patients. Including 11 patients with possible nonadherence (device refused or not returned) increased the nonadherence rate to 31 out of 56 (55\%) patients. Severe asthma criteria were fulfilled by 59 out of 69 patients. 47 were eligible for novel therapies, with confirmed nonadherence in 16 out of $32(50 \%)$ patients with EMD data; including seven patients with possible nonadherence increased the nonadherence rate to 23 out of 39 (59\%).

At least half the patients eligible for novel therapies were nonadherent to preventers. Nonadherence was often undetectable by clinical assessments. Preventer adherence must be confirmed objectively before employing novel severe asthma therapies.

Received: June 022017 | Accepted after revision: Feb 192018

Conflict of interest: M. Hew received an unrestricted grant from GlaxoSmithKline (GSK) during the conduct of the study to purchase adherence monitoring devices (GSK had no role or input into the design, conduct, analysis or reporting of the study); and has received grants from AstraZeneca (unrestricted grant to develop an electronic clinic template) and Novartis (unrestricted grant to host a severe asthma preceptorship), personal fees for advisory board participation from GSK, Seqirus and AstraZeneca; and has undertaken contracted research on behalf of AstraZeneca, Novartis, GSK and Sanofi, outside the submitted work; all for which his employer Alfred Health has been reimbursed.

Support statement: The authors acknowledge an unrestricted grant from GlaxoSmithKline (GSK) to purchase the electronic monitoring devices. GSK did not contribute to the design, conduct, analysis or reporting of this study. Funding information for this article has been deposited with the Crossref Funder Registry.

Copyright OERS 2018. This version is distributed under the terms of the Creative Commons Attribution NonCommercial Licence 4.0. 


\section{Introduction}

"Difficult asthma" is a term referring to patients who remain uncontrolled despite treatment at Steps 4 and 5 of the Global Initiative for Asthma (GINA) guidelines [1]. The overall prevalence of difficult asthma has been estimated at between $10 \%$ and $17 \%$ of asthma patients $[1,2]$.

A major contributor to difficult asthma is unsuppressed airway inflammation consequent to relative corticosteroid insensitivity [3]. In such patients, novel treatment options are now licensed for specific disease phenotypes, specifically monoclonal biologics targeting $\operatorname{IgE}$ (omalizumab) and the interleukin (IL)-5 pathways (mepolizumab, reslizumab) or bronchial thermoplasty [4-7]. Such treatments are expensive and should only be considered after standard therapy (including high-dose inhaled corticosteroids (ICSs), usually in combination with a long-acting bronchodilator) has been optimised [8-11].

Medication adherence can be defined as "the degree to which the medication use of the patient corresponds to the prescribed regimen" $[12,13]$. Patient nonadherence to medications can thus vary over time, and can be both intentional (e.g. due to fear of side-effects) and nonintentional (e.g. due to cost or forgetfulness). Identifying and addressing nonadherence to inhaled respiratory medications has been identified as an urgent priority for international policy makers [14]. Medication nonadherence is particularly prevalent in difficult asthma, with previous estimates of nonadherence of $\sim 50 \%$ by prescription refills [15-17]. Assessing prescription refills can be challenging if there are multiple prescribers and dispensing pharmacies. In clinical practice, preventer adherence is usually assessed subjectively by the treating health professional or based on patient self-report, which are both notoriously inaccurate [18]. However, inaccurate subjective assessments can have significant consequences: a large proportion of patients with difficult asthma also have severe biological asthma and poorer clinical outcomes are seen in those who are nonadherent to inhaled preventers [19]. Additionally, if nonadherence remains undetected in difficult asthma, patients may proceed inappropriately to targeted biological therapy or thermoplasty [9].

Detailed objective measurements of adherence may now be obtained by electronic monitoring devices (EMDs) [20-22]. These devices can be placed on the patient's preventer inhaler on initial contact and data downloaded at the next clinic visit. EMDs have been used to examine nonadherence in asthma, but data regarding their utility in difficult asthma are limited.

We hypothesised that nonadherence in difficult asthma remains a significant issue in the era of novel severe asthma therapies. We used an EMD to objectively measure preventer nonadherence in difficult asthma and compared this with structured, albeit subjective, clinician assessment. We specifically examined the rate of nonadherence among patients suitable for anti-IgE and anti-IL-5 biologics or thermoplasty.

\section{Materials and methods}

Patients were referred with difficult asthma if their treating respiratory or allergy specialists had difficulty with their asthma management. Reasons for referral (usually multiple) were diagnostic dilemma, poor symptom control, frequent or severe exacerbations, poor lung function, or patient factors, including comorbidities and suspected nonadherence [23].

Patients undergo systematic evaluation over 6 months in three visits to: confirm the diagnosis of severe asthma, to identify and address exacerbating triggers or comorbidities (including anxiety and depression, vocal cord dysfunction, dysfunctional breathing, gastro-oesophageal reflux disease, obstructive sleep apnoea, allergic rhinitis, and chronic rhinosinusitis), and to determine the inflammatory phenotype to facilitate selection of optimal medical therapy, including targeted biologics [24]. As previously described, the assessment process is supported by questionnaires for the assessment of the patient's asthma control (Asthma Control Test (ACT)) [25] and quality of life (Asthma Quality of Life Questionnaire (AQLQ)) [26], as well as a comorbidity questionnaire battery, an electronic platform and a panel discussion for each patient [27-29]. Permission was obtained for all administered questionnaires.

Our centre prescribes asthma biologics [30], but does not perform bronchial thermoplasty.

This study included consecutive patients who entered the difficult asthma protocol between May 1, 2015 and December 31, 2016. The study was approved by the Alfred Health Ethics Committee (285/15).

\section{Eligibility for novel asthma therapies}

For this study, patients were categorised as eligible for biologics if they met American Thoracic Society (ATS)/European Respiratory Society (ERS) criteria for severe and uncontrolled asthma [24], and had either an eosinophilic (blood eosinophil count $\geqslant 0.3 \times 10^{9} \mathrm{~L}^{-1}$; suitable for anti-IL-5 therapy) or allergic phenotype (serum IgE level $\geqslant 30 \mathrm{kU} \cdot \mathrm{mL}^{-1}$ and sensitisation to an aeroallergen based on skin testing or serum-specific IgE; suitable for anti-IgE therapy). 
Patients were categorised as eligible for thermoplasty based on entry criteria for the Research in Severe Asthma (RISA) trial [7]; high-dose ICS/LABA inhalers, pre-bronchodilator forced expiratory volume in $1 \mathrm{~s}$ $>50 \%$ predicted, positive bronchoprovocation or bronchodilator response, uncontrolled asthma and no current smoking nor prior smoking history of $\geqslant 10$ pack-years.

\section{Adherence assessment}

At the first clinic visit, the difficult asthma protocol physician would explain to each patient that the routine procedure for all patients was to provide an EMD for objective monitoring of asthma management if their inhaler device was compatible. Language was aimed to be neutral and nonjudgemental. A Smartinhaler device (Adherium, Auckland, New Zealand) was attached to the patient's preventer inhaler. EMDs were available for a variety of ICS or ICS/long-acting $\beta$-agonist (LABA) combination inhalers, specifically metered dose inhalers and dry powder devices for Flixotide (fluticasone propionate), Seretide (salmeterol and fluticasone propionate), Pulmicort (budesonide) and Symbicort (budesonide and formoterol). EMDs were not available for other ICS or ICS/LABA combination inhalers. The EMD was able to record the timing of the doses taken according to the number prescribed for morning or evening.

Data were downloaded at the scheduled follow-up visit at the 2-month time-point. Data collected included the date, time and number of actuations, preventing "dose dumping".

For the purposes of this study, the patient was considered adherent if $>75 \%$ of prescribed doses were actuated at the times they were prescribed, based on an increased risk of exacerbations reported below this cut-point [31]. Day-to-day adherence rate was not reported. Patients were defined as possibly nonadherent if they declined to have the device added to their inhaler or did not return the device despite reminders.

Adherence was also assessed at the first visit by the referring specialist, the patient, the difficult asthma clinic respiratory physician and a clinical asthma nurse. Referring specialists also completed a standardised referral form and could indicate whether they felt the patient was nonadherent.

At the first clinic visit during systematic evaluation, self-reported adherence was documented if the patient agreed with the following statement "I follow my medication plan".

The difficult asthma clinic specialist assessed patient adherence using the components of the validated Adult Asthma Adherence Questionnaire [32], including specific questions surrounding forgetfulness, a perception that preventer treatment was unnecessary, fear of side-effects and cost. Following the assessment, physicians were prompted to estimate inhaler adherence as $<50 \%, 50-75 \%$ or $>75 \%$.

At the first clinic visit, all patients underwent clinical asthma nurse assessment and education to address the patient's understanding of asthma, inhaler technique and adherence. The asthma nurse fitted the electronic device to the patient's inhaler. Following assessment, nursing staff were prompted to estimate adherence as "good", "partial" or "poor". Patients estimated to have "partial" or "poor" adherence were considered nonadherent.

\section{Statistical analysis}

Data analysis was performed using SPSS version 22 (IBM, Armonk, NY, USA). Categorical variables are presented as percentages (frequency) and continuous variables are presented as mean or median values with standard deviation and/or range. T-test for comparison of means and Fisher's exact or Chi-squared tests for comparison of proportions were performed where appropriate.

\section{Results}

During the study period, 71 consecutive patients underwent systematic evaluation. Two patients did not have asthma, had their inhalers discontinued and were excluded from further analysis. Baseline characteristics of the remaining 69 patients are presented in table 1.

In this difficult asthma cohort, poor asthma control and quality of life were reflected in the average ACT and AQLQ scores. $86 \%$ of patients were on GINA Step 4 or 5 asthma treatment. 59 out of 69 (85\%) patients had severe asthma, all of whom had uncontrolled asthma (as defined by the ERS/ATS guidelines) [24].

\section{Eligibility for biologics and thermoplasty}

$30(43.5 \%)$ patients were eligible for anti-IgE therapy and 22 (31.9\%) for anti-IL-5 therapy, with 38 (55\%) potential candidates for either. 26 patients (37.7\%) were eligible for thermoplasty. In total, $47(68 \%)$ patients were eligible for a biologic or thermoplasty, or both (figure 1).

EMD adherence assessment

69 patients were considered for an EMD. The flow of patients through each stage of the study is shown in figure 2 . 
Adherence outcomes for all 69 difficult asthma patients are shown in figure 3a. "Unknown adherence": adherence status could not be objectively assessed in 13 patients; 10 did not use a preventer with a compatible EMD and three returned a device which malfunctioned. "Possible nonadherence": another 11 exhibited behaviour suggestive of nonadherence; two patients refused the EMD and nine did not return the device, some of whom reported the device as lost. "Confirmed nonadherence": of 45 patients who returned a device with usable data, $20(44.4 \%)$ were nonadherent; mean \pm SD rate of nonadherence was $51.4 \pm 20.8 \%$ (interquartile range (IQR) 38-65\%). "Confirmed adherence": 25 out of 45 (55.6\%) patients had documented preventer adherence of $>75 \%$; mean \pm SD rate of adherence was $89 \pm 6.3 \%$ (IQR $84-95 \%$ ).

If patients with confirmed and possible (those who refused or did not return the EMD) nonadherence were grouped together, the overall nonadherence rate increased to 31 out of 56 (55.3\%) patients.

Confirmed nonadherent patients had higher mean serum eosinophils $\left(0.42 \times 10^{9}\right.$ versus $0.22 \times 10^{9} \mathrm{~L}^{-1}$; $\mathrm{p}<0.05)$ than confirmed adherent patients. There were no other significant associations of other clinical features that differed between adherent and nonadherent patients (table 2). The mean ICS dose ( $\mu \mathrm{g}$ fluticasone equivalent) was not significantly different in patients who were adherent $(982 \mu \mathrm{g})$ or nonadherent $(850 \mu \mathrm{g})$.

\section{TABLE 1 Baseline characteristics}

\begin{tabular}{|c|c|}
\hline Subjects & 69 \\
\hline Age years & $52 \pm 14.2(19-76)$ \\
\hline Female & $41(59.4)$ \\
\hline \multicolumn{2}{|l|}{ Smoking status } \\
\hline Never-smoker & 46 (66.7) \\
\hline Ex-smoker & 22 (31.9) \\
\hline Current smoker & $1(1.4)$ \\
\hline $\mathrm{BMI} \mathbf{k g} \cdot \mathrm{m}^{-2}$ & $30 \pm 6.9$ \\
\hline Early-onset asthma $<18$ years & $34(49.3)$ \\
\hline Pre-bronchodilator FEV 1 \% pred & $62 \pm 20.2$ \\
\hline Change in FEV1 \% pred following bronchodilator & $14.2 \pm 15$ \\
\hline FEV $/$ FVC $\%$ & $61 \pm 15.4$ \\
\hline Airflow obstruction at baseline (FEV $1<80 \%, \mathrm{FEV}_{1} / \mathrm{FVC}<70 \%$ ) & $41(59.4)$ \\
\hline Variable airflow obstruction demonstrable & $61(88.4)$ \\
\hline$\geqslant 12 \%$ and $\geqslant 200 \mathrm{~mL}$ improvement in $\mathrm{FEV} 1$ following bronchodilator & $47(77)$ \\
\hline$>12 \%$ variability in peak flow charting over 2 weeks & $12(19.7)$ \\
\hline Positive bronchial provocation challenge test with mannitol & 2 (3.3) \\
\hline Blood eosinophils $\times 10^{9} \mathrm{~L}^{-1}$ & $0.33 \pm 0.33(0-1.73)$ \\
\hline Eosinophils $\geqslant 0.3 \times 10^{9} \mathrm{~L}^{-1}$ & $28(40.6)$ \\
\hline FENO ppb & $36 \pm 31.2(4-137)$ \\
\hline $\operatorname{lgE} \mathrm{kU} \cdot \mathrm{L}^{-1}$ & $524 \pm 1006(2-4880)$ \\
\hline Atopic ${ }^{\#}$ & $47(68.1)$ \\
\hline ACT score & $13.6 \pm 5.19$ \\
\hline AQLQ score ${ }^{+}$ & $4.19 \pm 1.4$ \\
\hline $\begin{array}{l}\text { Exacerbations in last } 12 \text { months requiring oral ( }>3 \text { days or increase in } 20 \mathrm{mg} \text { from } \\
\text { baseline prednisolone dose) or intravenous corticosteroids }\end{array}$ & $5 \pm 4.7(0-30)$ \\
\hline \multicolumn{2}{|l|}{ Frequency of asthma exacerbations in 12 months } \\
\hline 0 & $4(5.8)$ \\
\hline 1 & $8(11.6)$ \\
\hline 2 & $2(15.9)$ \\
\hline$\geqslant 3$ & $46(66.7)$ \\
\hline On ICS/LABA combination & 66 (95.7) \\
\hline Total ICS dose $\mu \mathrm{g}$ fluticasone equivalent & $992 \pm 538(0-3200)$ \\
\hline On OCSs & $17(24.6)$ \\
\hline Total OCS dose $\mathrm{mg}$ & $8.7 \pm 6.23(1-25)$ \\
\hline Severe asthma by ATS/ERS guidelines & $59(85.5)$ \\
\hline Anxiety and depression ${ }^{\S}$ & $24(35)$ \\
\hline
\end{tabular}

Data are presented as $n$, mean \pm SD (range), mean \pm SD or $n(\%)$. BMI: body mass index; FEV1: forced expiratory volume in $1 \mathrm{~s}$; FVC: forced vital capacity; FENO: exhaled nitric oxide fraction; ACT: Asthma Control Test; AQLQ: Asthma Quality of Life Questionnaire; ICS: inhaled corticosteroid; LABA: long-acting $\beta$-agonist; OCS: oral corticosteroid; ATS: American Thoracic Society; ERS: European Respiratory Society. \#: positive skin prick test or serum-specific IgE to commonly tested aeroallergens; " : < 15 indicating poor control; ${ }^{+}$: out of 7, high score indicating better quality of life; ${ }^{\S}$ : diagnosis based on presence of clinical symptoms and Hospital Anxiety and Depression Scale [33] score $\geqslant 11$ or known history on treatment. 
FIGURE 1 Patients eligible for novel asthma therapies: biologics and bronchial thermoplasty $(47$ out of 69). IL: interleukin.
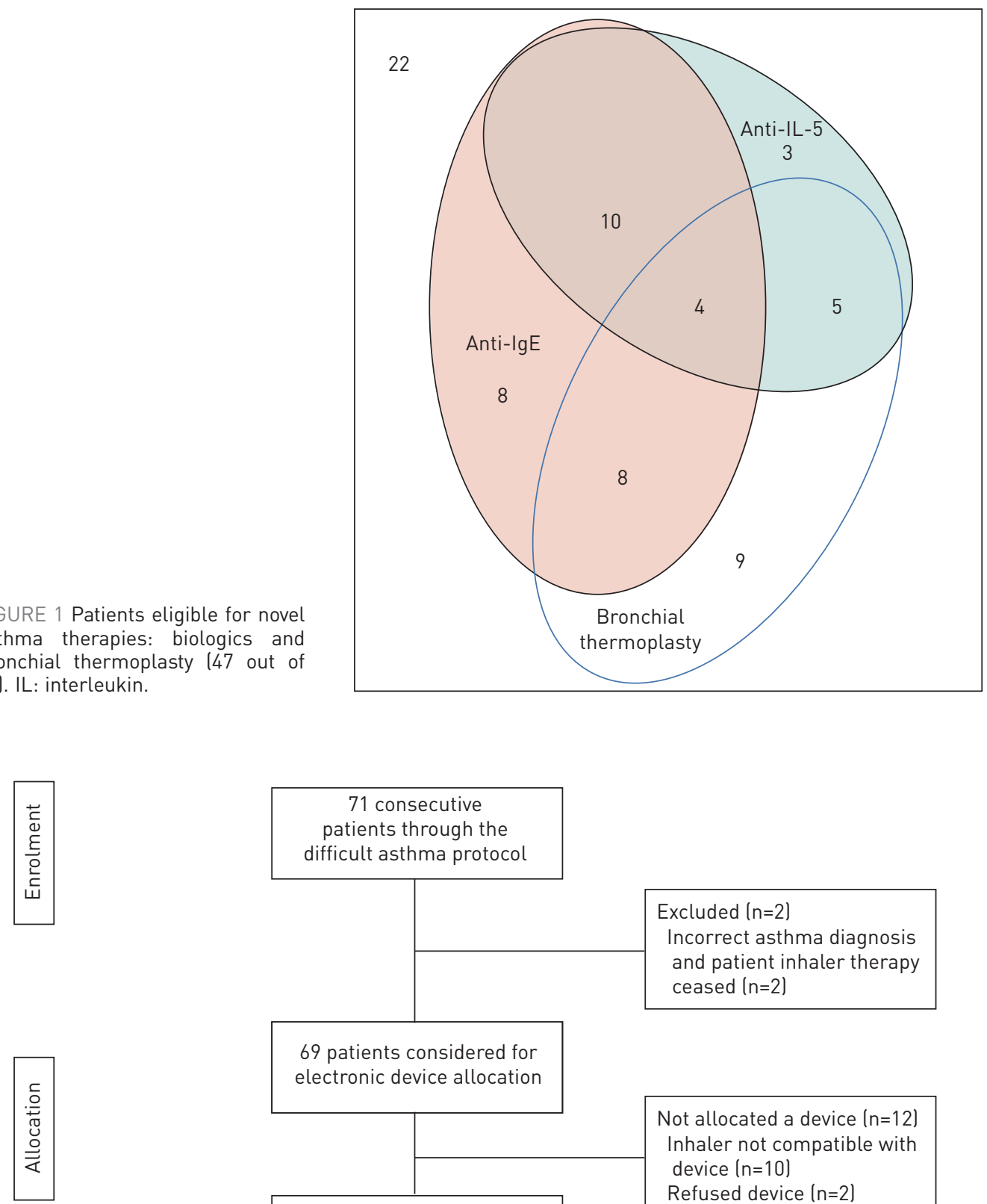

69 patients considered for electronic device allocation

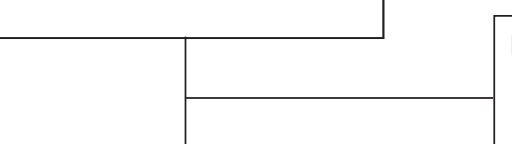

Not allocated a device $(\mathrm{n}=12)$ Inhaler not compatible with device ( $n=10$ )

Refused device ( $n=2)$

57 patients allocated electronic monitoring device
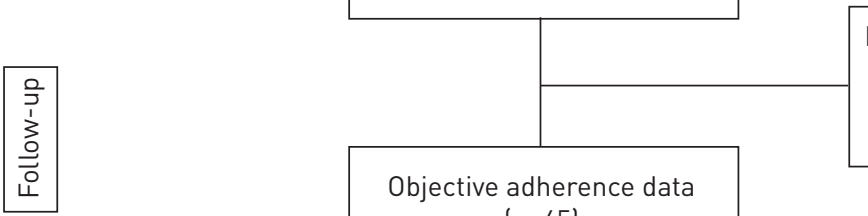

No usable data $(n=12)$

Device malfunction ( $\mathrm{n}=3$ )

Device reported lost or not returned $(n=9)$

\section{Objective adherence data} $(n=45)$

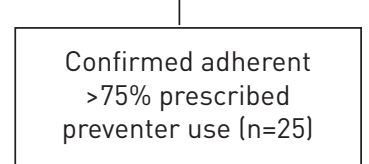

Confirmed nonadherent

$\leqslant 75 \%$ prescribed

preventer use $(\mathrm{n}=20)$

FIGURE 2 Patient flow through each stage of the study. 
a)

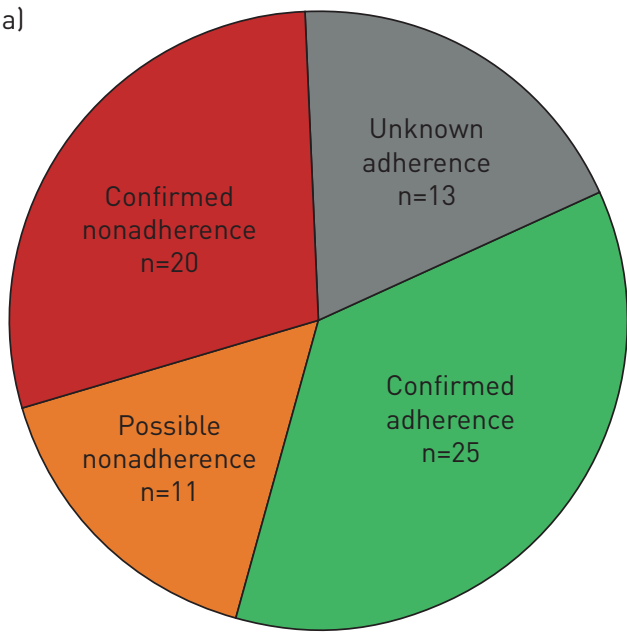

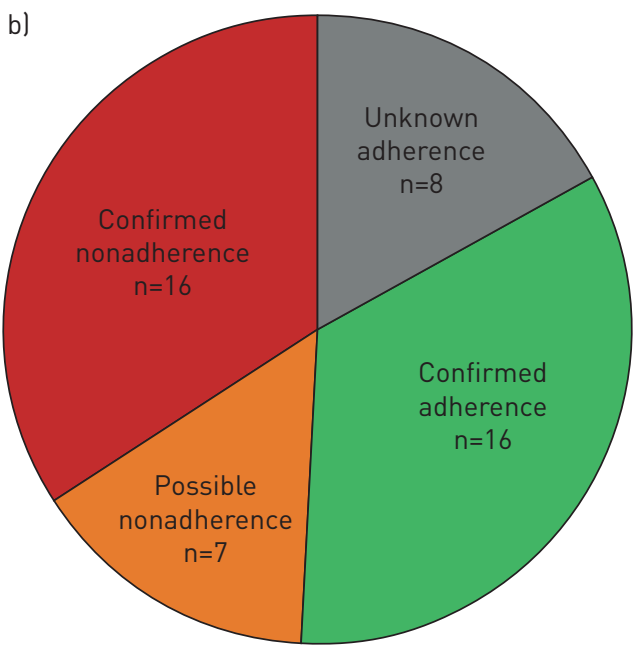

FIGURE 3 Patients with unknown adherence, confirmed adherence, possible nonadherence and confirmed nonadherence in a) all 69 difficult asthma patients and b) the 47 patients eligible for novel asthma therapies (biologics or thermoplasty).

Among 47 patients either eligible for biologics or bronchial thermoplasty, adherence could not be assessed in eight (EMD-incompatible inhaler or device malfunction) and nonadherence was possible in seven (refused or did not return the EMD). 32 patients returned usable EMD data, of whom 16 out of $32(50 \%)$ had confirmed nonadherence on EMD assessment. Combining confirmed and possible nonadherent populations gave a nonadherence rate among those eligible for novel therapies of 23 out of 39 (59\%) patients (figure $3 b$ ).

TABLE 2 Characteristics of adherent compared with nonadherent patients

\begin{tabular}{|c|c|c|c|}
\hline & Adherent & Nonadherent & p-value \\
\hline Subjects & 25 & 20 & \\
\hline Age years & $54 \pm 12$ & $54 \pm 16$ & NS \\
\hline Female & $13(52)$ & $11(55)$ & NS \\
\hline \multicolumn{4}{|l|}{ Smoking status } \\
\hline Never-smoker & $14(56)$ & $14(70)$ & \\
\hline Ex-smoker & $11(44)$ & $5(25)$ & \\
\hline Current smoker & $0(0)$ & $1(5)$ & \\
\hline BMI $\mathrm{kg} \cdot \mathrm{m}^{-2}$ & $30 \pm 5$ & $31 \pm 8$ & NS \\
\hline Early-onset asthma $<18$ years & $13(52)$ & $8(40)$ & NS \\
\hline Pre-bronchodilator FEV $1 \%$ pred & $65 \pm 22$ & $60 \pm 18$ & NS \\
\hline FEV $/$ FVC $\%$ & $61 \pm 17$ & $58 \pm 12$ & NS \\
\hline Airflow obstruction at baseline (FEV $1<80 \%, \mathrm{FEV}_{1} / \mathrm{FVC}<70 \%$ ) & $14(56)$ & $13(65)$ & NS \\
\hline Blood eosinophils $\times 10^{9} \mathrm{~L}^{-1}$ & $0.22 \pm 0.21$ & $0.42 \pm 0.34$ & $<0.05$ \\
\hline FENO ppb & $27.22 \pm 18$ & $41.4 \pm 30$ & NS \\
\hline $\operatorname{lgE~kU} \cdot \mathrm{L}^{-1}$ & $369.5 \pm 736$ & $551.5 \pm 1030$ & NS \\
\hline ACT score $\#$ & $12.2 \pm 4$ & $13.5 \pm 6$ & NS \\
\hline$A Q L Q$ score $\pi$ & $4.34 \pm 1$ & $3.99 \pm 1$ & NS \\
\hline $\begin{array}{l}\text { Exacerbations in last } 12 \text { months requiring oral ( }>3 \text { days or } \\
\text { increase in } 20 \mathrm{mg} \text { from baseline prednisolone dose) or } \\
\text { intravenous corticosteroids }\end{array}$ & $3.5 \pm 18$ & $2.8 \pm 2$ & NS \\
\hline Total ICS dose $\mu \mathrm{g}$ fluticasone equivalent & $982 \pm 444$ & $850 \pm 379$ & NS \\
\hline Total OCS dose $\mathrm{mg}$ & $4 \pm 2$ & $9.4 \pm 5$ & NS \\
\hline Severe asthma by ATS/ERS guidelines & $21(84)$ & $19(95)$ & NS \\
\hline Anxiety or depression ${ }^{+}$ & $11(44)$ & $7(35)$ & NS \\
\hline
\end{tabular}

Data are presented as $n$, mean \pm SD or $n(\%)$. BMI: body mass index; FEV1: forced expiratory volume in $1 \mathrm{~s}$; FVC: forced vital capacity; FENO: exhaled nitric oxide fraction; ACT: Asthma Control Test; AQLQ: Asthma Quality of Life Questionnaire; ICS: inhaled corticosteroid; OCS: oral corticosteroid; ATS: American Thoracic Society; ERS: European Respiratory Society; NS: nonsignificant. \#: $<15$ indicating poor control; П: out of 7 , high score indicating better quality of life; ${ }^{+}$: diagnosis based on presence of clinical symptoms and Hospital Anxiety and Depression Scale [33] score $\geqslant 11$ or known history on treatment. 


\section{Subjective adherence assessment}

Two of the 45 patients with EMD data had nonadherence identified by the referring specialist as a reason for the patient's poor asthma control. Two patients admitted that they did not follow their prescribed medication plan. Protocol physicians identified five patients as being nonadherent (of these, two were proven to be adherent objectively), whereas asthma nurses identified seven patients as being nonadherent (of these, two were also proven to be adherent objectively) (figure 4).

Compared with the EMD, the sensitivity and specificity of physician detection of nonadherence was $15 \%$ (95\% CI 3.2-37.9\%) and 92\% (95\% CI 74-99\%), respectively. The sensitivity and specificity of asthma nurse assessment was $27.8 \%$ (95\% CI 9.7-53.5\%) and 91.67\% (95\% CI 73-99\%), respectively (figure 4).

\section{Discussion}

Among patients otherwise suitable for novel severe asthma therapies, our study shows an alarmingly high rate of nonadherence. Furthermore, most cases of nonadherence remained undetected despite a series of subjective clinical assessments by the referring respiratory or allergy specialist, the difficult asthma protocol specialist and asthma nursing staff. This finding emphasises the indispensable value of assessing nonadherence objectively prior to initiating biologics or performing thermoplasty for severe asthma [9].

Previous studies have shown high rates of nonadherence among difficult asthma patients by monitoring prescription refills, which gives an indication of long-term medication use $[16,34,35]$. This can be difficult to perform in health systems such as our Australian setting, where patients may obtain preventers through multiple prescribers and at multiple dispensing pharmacies of their choice. Prescription refills and other indirect methods of measuring medication adherence such as canister weights also cannot confirm that a patient actually takes their medication at the correct time. We therefore chose to use EMDs, which provide detailed information on inhaler use.

The true prevalence of nonadherence in our difficult asthma population likely lies between $44 \%$ and $55 \%$, consistent with previous studies $[15,16]$. However, the finding of even greater nonadherence among patients suitable for biologics or thermoplasty supports the premise that nonadherence is intrinsically linked to more severe markers of disease. Indeed, we found that nonadherent patients had higher peripheral eosinophil counts. A previous study also found greater sputum eosinophilia among nonadherent patients [16]. Thus, an indication for instituting severe asthma biologics may also indicate a higher risk of nonadherence. Interestingly, severity of asthma symptoms, frequency of exacerbations or poorer lung function did not seem to influence rates of medication adherence. Similarly, prevalence of anxiety and depression was not increased among patients who were found to be nonadherent.

The optimal method to assess preventer adherence remains unclear and EMDs are not infallible. An initial pilot study of the device we used recorded a mean accuracy of $97 \%$ [20]. In a clinical trial of 303 patients incorporating extensive pre- and post-study checks, there was a $6.5 \%$ malfunction rate. In addition, $3.5 \%$ of devices were lost or thrown away by participants [36]. Other trials have reported higher malfunction rates of between $15 \%$ and $20 \%[37,38]$.

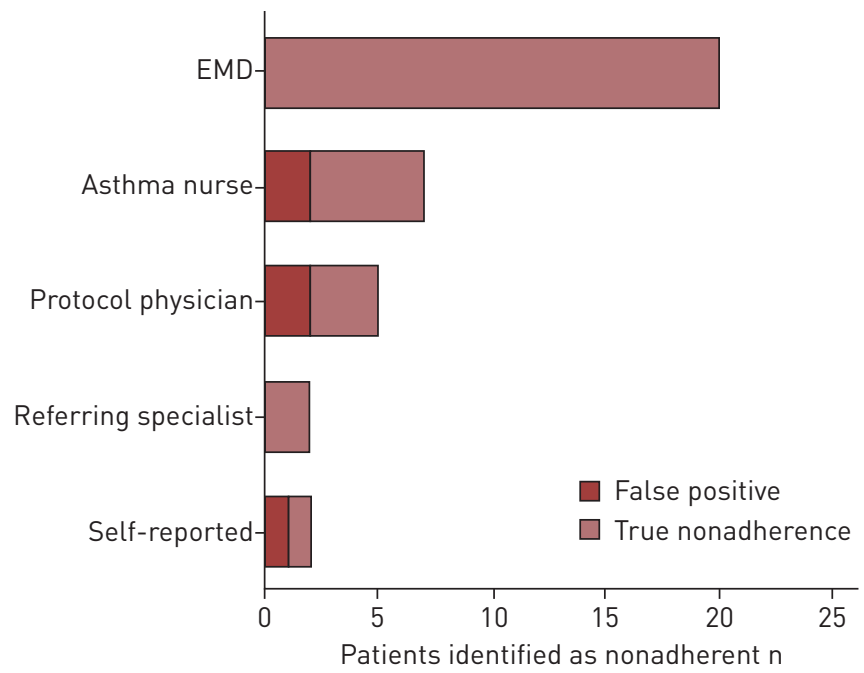

FIGURE 4 Detection of nonadherence by subjective methods in 45 difficult asthma patients with objective data from the electronic monitoring device (EMD). 
In our study, adherence could not be assessed objectively in $19 \%$ of patients either due to EMD-incompatible inhalers (14.4\%) or device malfunction (5.3\%). Patients possibly subverted the process in another 11 cases (16\%), either by refusing or not returning the device despite repeated requests; such behaviour has been reported in previous pragmatic studies [22] and in our view could also represent possible medication nonadherence. We acknowledge that some patients may have felt uncomfortable with being monitored and this may have influenced the acceptance of the device or failure-to-return rate. We chose not to implement financial incentives to return the device.

Consequently, objective EMD data was obtained in less than two-thirds of our cohort. The failure-to-return rate is higher than in previously reported clinical trials, reflecting the challenges of real-life evaluation of consecutive clinical patients, which is always more difficult than when participants have been selected for a trial. Due to cost, some guidelines have questioned the utility of EMDs in the management of asthma, outside of the research setting [39]. The EMDs cost approximately USD150 (AUD200) in 2018 prices, but this could be considered trivial in comparison with the cost of severe asthma biologics or thermoplasty procedures. However, the true cost of monitoring does go beyond the cost of devices, such as the costs of time required to manage these in the clinic setting: education of the patient, testing to reduce malfunction rates and the efforts required to ensure their return.

Self-reporting of nonadherence was unreliable and poorly sensitive in our cohort, consistent with previous studies [40, 41]. Subjective clinical assessments were also poorly sensitive for detecting EMD-confirmed nonadherence. This was despite assessment by an expert and experienced difficult asthma service, multiple assessments by three health professionals (referring physician, treating physician and asthma nurse), and the use of standardised assessments including a validated adherence tool [32]. In light of this, objective assessments are clearly indispensable to adequately detect nonadherence. Interestingly, a minority of patients who were deemed poorly adherent by clinical assessment were subsequently proven to be adherent by electronic monitoring. Although described as "false positives", this cohort may have improved their adherence behaviour in the knowledge that they were being monitored. Nevertheless, these patients may have had their access to advanced therapies inappropriately limited by the healthcare team if reliance had been placed solely on subjective measurement of adherence.

$25 \%$ of our patient cohort was on oral steroids. It could be argued that patients with uncontrolled asthma despite oral steroids may require a novel therapy such as a biologic irrespective of their adherence to inhaled steroids. However, we would argue that patients nonadherent to inhaled therapy would also be likely to be nonadherent to oral medication [15]. (Assessing adherence to oral corticosteroids was beyond the scope of this study due to nonavailability of in-house serum prednisolone levels.) In addition, it is a government funding body requirement in Australia that adherence to inhaled steroids is documented prior to prescription of biologics.

The aim of our study was to detect nonadherence, not to manage it, and this study was not designed to report on longitudinal outcomes. However, an EMD can be used to provide feedback and deliver an audio reminder to the patient. These measures have been shown to improve adherence [21] and, in a paediatric population, led to fewer exacerbations requiring oral corticosteroids or hospitalisation [22]. Such benefits have yet to be shown in a difficult asthma population and would be an interesting area for future research. The overall outcomes of our 6-month, three-visit systematic assessment protocol have been previously reported [29].

This study was conducted at a single centre with an interest in difficult asthma, so the generalisability of our findings is unknown. However, the rate of nonadherence in our cohort is consistent with those reported from other difficult asthma centres in other health systems $[15,16]$. Nonadherence in nonsevere asthma is even more prevalent. In a cross-sectional community study of patients with asthma (over half of which were "well controlled"), $65 \%$ of respondents were nonadherent to a preventer $<4$ days a week [42]. For this study, we defined eligibility for severe asthma biologics based on generic criteria and suitability for thermoplasty based on inclusion criteria for the single randomised RISA trial of thermoplasty in severe asthma [7]. However, additional criteria may apply according to local licensing authorities and funding arrangements. We further acknowledge that nonadherent patients identified by EMD may be a heterogeneous group that we were unable to stratify further in this study. Patients taking $<75 \%$ of prescribed doses could be described as partially adherent and probably represent a diverse group of patients. However, this cut-off was chosen because published data demonstrates such patients are at an increased risk of adverse asthma outcomes [31]. While EMDs can confirm that an inhaler is actuated, they cannot determine if the patient actually inhaled the medication nor whether inhaler technique was satisfactory. Nevertheless, we maintain that EMD-confirmed nonadherence is a robust finding. The provision of the EMD alerted patients that their adherence was being monitored. It is possible that the degree of nonadherence might have been even greater had patients been unaware of monitoring, i.e. the Hawthorne effect [43]. 
We conclude that preventer nonadherence in difficult asthma remains disturbingly high in the era of novel (and expensive) therapies for severe asthma. There are a multitude of factors that may underlie nonadherence and we advocate for further research to be carried out in this area. Subjective assessment of adherence is highly unreliable, so objective assessments are imperative prior to initiating severe asthma biologics or performing thermoplasty.

\section{Acknowledgements}

The authors gratefully acknowledge the invaluable assistance of Eddie Weber and Anita Hazard (The Alfred Hospital, Melbourne Australia) in the nursing assessment of the difficult asthma patients.

Author contributions: Conception and design: J. Lee, M. Hew, T.R. Tay, N. Radhakrishna, R. Hoy, E. Dabscheck and R. O'Hehir. Data acquisition, analysis and interpretation: J. Lee, M. Hew, T.R. Tay, A. Mackay, F. Hore-Lacy, R. Hoy and E. Dabscheck. Drafting the manuscript for important intellectual content: J. Lee, M. Hew, T.R. Tay, N. Radhakrishna, R. Hoy, E. Dabscheck, F. Hore-Lacy, A. Mackay and R. O'Hehir. Approval of final manuscript for submission: J. Lee, M. Hew, T.R. Tay, N. Radhakrishna, R. Hoy, E. Dabscheck, R. O’Hehir, F. Hore-Lacy and A. Mackay. J. Lee and M. Hew had full access to all the data in the study and take responsibility for the integrity of the data and the accuracy of the data analysis, including and especially any adverse effects.

\section{References}

1 Reddel HK, Bateman ED, Becker A, et al. A summary of the new GINA strategy: a roadmap to asthma control. Eur Respir J 2015; 46: 622-639.

2 Hekking P-PW, Wener RR, Amelink M, et al. The prevalence of severe refractory asthma. J Allergy Clin Immunol 2015; 135: 896-902.

3 Hew M, Chung KF. Corticosteroid insensitivity in severe asthma: significance, mechanisms and aetiology. Intern Med J 2010; 40: 323-334.

4 Bousquet J, Siergiejko Z, Świebocka E, et al. Persistency of response to omalizumab therapy in severe allergic (IgE-mediated) asthma. Allergy 2011; 66: 671-678.

5 Ortega HG, Liu MC, Pavord ID, et al. Mepolizumab treatment in patients with severe eosinophilic asthma. $N$ Engl J Med 2014; 371: 1198-1207.

6 Castro M, Zangrilli J, Wechsler ME, et al. Reslizumab for inadequately controlled asthma with elevated blood eosinophil counts: results from two multicentre, parallel, double-blind, randomised, placebo-controlled, phase 3 trials. Lancet Respir Med 2015; 3: 355-366.

7 Pavord ID, Cox G, Thomson NC, et al. Safety and efficacy of bronchial thermoplasty in symptomatic, severe asthma. Am J Respir Crit Care Med 2007; 176: 1185-1191.

8 Draikiwicz S, Oppenheimer J. Use of biological agents in asthma. Chest 2017; 151: 249-251.

9 Barnes PJ. Counterpoint: will new anti-eosinophilic drugs be useful in asthma management? No. Chest 2017; 151: $17-20$.

10 Whittington MD, McQueen RB, Ollendorf DA, et al. Assessing the value of mepolizumab for severe eosinophilic asthma: a cost-effectiveness analysis. Ann Allergy Asthma Immunol 2016; 118: 220-225.

11 Zafari Z, Sadatsafavi M, Marra CA, et al. Cost-effectiveness of bronchial thermoplasty, omalizumab, and standard therapy for moderate-to-severe allergic asthma. PLoS One 2016; 11: e0146003.

12 Vrijens B, Dima AL, Van Ganse E, et al. What we mean when we talk about adherence in respiratory medicine. J Allergy Clin Immunol Pract 2016; 4: 802-812.

13 World Health Organization. Adherence to long-term therapies: evidence for action. 2003. www.who.int/chp/ knowledge/publications/adherence_full_report.pdf Date last accessed: February 23, 2018.

14 van Boven JFM, Lavorini F, Dekhuijzen PNR, et al. Urging Europe to put non-adherence to inhaled respiratory medication higher on the policy agenda: a report from the First European Congress on Adherence to Therapy. Eur Respir J 2017; 49: 1700076.

15 Gamble J, Stevenson M, McClean E, et al. The prevalence of nonadherence in difficult asthma. Am J Respir Crit Care Med 2009; 180: 817-822.

16 Murphy AC, Proeschal A, Brightling CE, et al. The relationship between clinical outcomes and medication adherence in difficult-to-control asthma. Thorax 2012; 67: 751-753.

17 Boulet L-P, Vervloet D, Magar Y, et al. Adherence. Clin Chest Med 2012; 33: 405-417.

18 Patel M, Perrin K, Pritchard A, et al. Accuracy of patient self-report as a measure of inhaled asthma medication use. Respirology 2013; 18: 546-552.

19 Williams LK, Pladevall M, Xi H, et al. Relationship between adherence to inhaled corticosteroids and poor outcomes among adults with asthma. J Allergy Clin Immunol 2004; 114: 1288-1293.

20 Foster JM, Smith L, Usherwood T, et al. The reliability and patient acceptability of the SmartTrack device: a new electronic monitor and reminder device for metered dose inhalers. J Asthma 2012; 49: 657-662.

21 Chan AHY, Stewart AW, Harrison J, et al. The effect of an electronic monitoring device with audiovisual reminder function on adherence to inhaled corticosteroids and school attendance in children with asthma: a randomised controlled trial. Lancet Respir Med 2015; 3: 210-219.

22 Morton RW, Elphick HE, Rigby AS, et al. STAAR: a randomised controlled trial of electronic adherence monitoring with reminder alarms and feedback to improve clinical outcomes for children with asthma. Thorax 2016; 72: 347-354.

23 Radhakrishna N, Tay TR, Hore-Lacy F, et al. Profile of difficult to treat asthma patients referred for systematic assessment. Respir Med 2016; 117: 166-173.

24 Chung KF, Wenzel SE, Brozek JL, et al. International ERS/ATS guidelines on definition, evaluation and treatment of severe asthma. Eur Respir J 2014; 43: 343-373.

25 Nathan RA, Sorkness CA, Kosinski M, et al. Development of the asthma control test: a survey for assessing asthma control. J Allergy Clin Immunol 2004; 113: 59-65.

26 Juniper EF, Guyatt GH, Epstein RS, et al. Evaluation of impairment of health related quality of life in asthma: development of a questionnaire for use in clinical trials. Thorax 1992; 47: 76-83. 
Gibson PG Reddel H, McDonald VM, et al. Effectiveness and response predictors of omalizumab in a seve allergic asthma population with a high prevalence of comorbidities: the Australian Xolair Registry. Intern Med $J$ 2016; 46: 1054-1062.

31 Williams LK, Peterson EL, Wells K, et al. Quantifying the proportion of severe asthma exacerbations attributable to inhaled corticosteroid nonadherence. J Allergy Clin Immunol 2011; 128: 1185-1191.

32 Schatz M, Zeiger RS, Yang S-J, et al. Development and preliminary validation of the Adult Asthma Adherence Questionnaire ${ }^{\mathrm{TM}}$. J Allergy Clin Immunol Pract 2013; 1: 280-288.

33 Zigmond AS, Snaith RP. The Hospital Anxiety and Depression Scale. Acta Psychiatr Scand 1983; 67: 361-370.

34 Robinson DS, Campbell DA, Durham SR, et al. Systematic assessment of difficult-to-treat asthma. Eur Respir J 2003; 22: 478-483.

35 Heaney LG, Horne R. Non-adherence in difficult asthma: time to take it seriously. Thorax 2011; 67: 268-270.

36 Patel M, Pilcher J, Travers J, et al. Use of metered-dose inhaler electronic monitoring in a real-world asthma randomized controlled trial. J Allergy Clin Immunol Pract 2013; 1: 83-91.

37 Apter AJ, Wang X, Bogen DK, et al. Problem solving to improve adherence and asthma outcomes in urban adults with moderate or severe asthma: a randomized controlled trial. J Allergy Clin Immunol 2011; 128: 516-523.

38 Rand C, Bilderback A, Schiller K, et al. Adherence with montelukast or fluticasone in a long-term clinical trial: results from the Mild Asthma Montelukast Versus Inhaled Corticosteroid Trial. J Allergy Clin Immunol 2007; 119: 916-923.

39 British Thoracic Society, Scottish Intercollegiate Guidelines Network. British guideline on the management of asthma. 2014. www.brit-thoracic.org.uk/document-library/clinical-information/asthma/btssign-asthma-guideline-2014 Date last accessed: February 23, 2018.

40 Krishnan JA, Bender BG, Wamboldt FS, et al. Adherence to inhaled corticosteroids: an ancillary study of the Childhood Asthma Management Program clinical trial. J Allergy Clin Immunol 2012; 129: 112-118.

41 Foster JM, Smith L, Bosnic-Anticevich SZ, et al. Identifying patient-specific beliefs and behaviours for conversations about adherence in asthma. Intern Med J 2012; 42: e136-e144.

42 Reddel HK, Sawyer SM, Everett PW, et al. Asthma control in Australia: a cross-sectional web-based survey in a nationally representative population. Med J Aust 2015; 202: 492-496.

43 Braunholtz DA, Edwards SJL, Lilford RJ. Are randomized clinical trials good for us (in the short term)? Evidence for a "trial effect". J Clin Epidemiol 2001; 54: 217-224. 